\title{
Valor da Queixa Clínica e Exame Físico no Diagnóstico da Incontinência Urinária
}

\author{
Role of Clinical History and Physical Examination in the \\ Diagnosis of Urinary Incontinence \\ Paulo Cezar Feldner Jr, Leonardo Robson P.S. Bezerra, Manoel João Bastista C. Girão, \\ Rodrigo Aquino de Castro, Marair Gracio F. Sartori, Edmund Chada Baracat, \\ Geraldo Rodrigues de Lima
}

\begin{abstract}
RESUMO
Objetivo: analisar a prevalência das queixas clinicas uroginecológicas correlacionando-as com o diagnóstico definitivo após o exame urodinâmico e comparar o sinal clínico de perda urinária com o estudo urodinâmico.

Métodos: foram analisadas, retrospectivamente, 114 pacientes atendidas no periodo de junho de 2000 a janeiro de 2001. Todas as pacientes foram avaliadas por meio de anamnese padronizada, exame fisico e estudo urodinâmico, sendo classificadas de acordo com o queixa clínica, presença do sinal de perda urinária durante o exame ginecológico e diagnóstico urodinâmico. Utilizou-se a análise estatística dos dados amostrais, por meio da determinação interna de um teste diagnóstico, para calcular a sensibilidade, especificidade e os valores preditivos positivo e negativo do sinal clínico.

Resultados: a média de idade foi de 51 anos (19-80), sendo que 61 encontravam-se no menacme (53,5\%) e 53 (46,5\%) na pós-menopausa. Destas, 10 (18,8\%) faziam uso da terapia de reposição hormonal. Do total de pacientes, 25 (21,9\%) haviam se submetido a cirurgias prévias para incontinência. A queixa de perda urinária isolada foi referida por 41 pacientes (36,0\%), a urgência/urge-incontinência isolada por 13 (11,4\%) e os sintomas mistos por 60 (52,6\%). Das pacientes com perda isolada, observou-se, à avaliação urodinâmica, que 34 (83\%) tinham incontinência urinária de esforço (IUE), nenhuma paciente apresentava instabilidade do detrusor (ID), 2 (4,9\%) incontinência urinária mista (IUM) e em 5 (12,1\%) o estudo foi normal. Daquelas com queixa de urgência/urge-incontinência isolada, observamos na avaliação urodinâmica que nenhuma tinha IUE, 5 (38,5\%) ID, 1 (7,7\%) IUM e em 7 (53,8\%) o estudo foi normal. Daquelas com sintomas mistos, identificamos na avaliação urodinâmica 25 com IUE (41,6\%), $10 \mathrm{com} I D(16,7 \%), 10$ IUM (16,7\%) e em 15 o estudo foi normal (25,0\%). O sinal clinico de perda ao exame fisico foi identificado em 50 (43,9\%) pacientes. Destas, 35 (70\%) tinham diagnóstico urodinâmico de IUE, 6 (12\%) IUE e outro diagnóstico e $9(18 \%)$ não tinham IUE. O sinal clinico estava ausente em 64 (56,1\%) mulheres. Destas, 23 $(35,9 \%)$ tinham diagnóstico urodinâmico de IUE, 7 (11\%) IUE e outro diagnóstico e 34 (53,1\%) não tinham IUE.

Conclusões: a história clinica associada ao exame fisico têm importância no manejo da incontinência urinária; porém, não devem ser utilizados como único critério para o diagnóstico. Os testes objetivos estão disponiveis e devem ser utilizados em conjunto com os dados clinicos.
\end{abstract}

PALAVRAS-CHAVE: Incontinência urinária. Exame clínico. Estudo urodinâmico.

Departamento de Ginecologia da Escola Paulista de Medicina - Setor de Uroginecologia e Cirurgia Vaginal Universidade Federal de São Paulo - EPM/UNIFESP

Correspondência: Paulo Cezar Feldner $\mathrm{Jr}$

Rua Machado Bitencourt, n ${ }^{\circ} 379$, apto 34 - Vila Clementino 04044-000 - São Paulo - SP

\section{Introdução}

Incontinência urinária é conceituada como toda condição na qual a perda involuntária de uri- 
na, objetivamente demonstrável, cause problema social ou higiênico à mulher ${ }^{1}$. Tem causa multifatorial, sendo elemento gerador de exclusão social, interferindo na saúde física e mental da paciente e comprometendo sua qualidade de vida.

O diagnóstico fisiopatológico é essencial, uma vez que incontinência de esforço pela deficiência esfincteriana ou pela hipermobilidade da uretra e a incontinência de urgência secundária à instabilidade do detrusor são as duas formas mais freqüentes de perda urinária. Sua diferenciação é prioridade antes de se propor um tratamento à paciente, especialmente quando há indicação de correção cirúrgica.

A anamnese deve conter alguns aspectos incluindo o início dos sintomas, sua duração, gravidade, condições associadas e descrição do impacto social e higiênico à mulher ${ }^{2}$. Embora a história forneça diversos dados, é freqüente não se obter o diagnóstico, uma vez que os sintomas urinários podem ser similares mesmo quando as etiologias são diferentes. Isto faz do exame fisico uma parte integrante da avaliação uroginecológica da paciente, constituindo, porém, apenas um segmento da avaliação ${ }^{3}$.

Farrar et al. ${ }^{4}$ observaram que pacientes com sintoma isolado de perda aos esforços usualmente têm bexigas estáveis, e o estudo urodinâmico não seria obrigatório nestas situações. Entretanto, vários pesquisadores relataram fraca correlação entre os sintomas e os achados urodinâmi$\cos ^{2,5-9}$, preconizando sua realização para a identificação das causas de incontinência.

O exame físico faz parte da rotina da investigação ginecológica da mulher incontinente visando reproduzir e caracterizar a incontinência, excluir distúrbios neurológicos, avaliar o suporte pélvico e excluir outras enfermidades pélvicas.

Alguns autores relataram que a história associada à demonstração clínica da perda urinária ao esforço é suficiente para o diagnóstico de incontinência urinária de esforço (IUE) ${ }^{10,11}$, porém poucos estudos investigaram a relação entre este sinal clínico e o diagnóstico urodinâmico final $^{12-14}$.

O objetivo deste trabalho foi analisar a prevalência das queixas clinicas uroginecológicas correlacionando-as com o diagnóstico urodinâmico final e comparar o sinal clínico de perda urinária com o estudo urodinâmico.

\section{Pacientes e Métodos}

Foram analisadas, retrospectivamente, 114 pacientes no período de junho de 2000 a janeiro de 2001, atendidas no setor de Uroginecologia e Cirurgia Vaginal da UNIFESP/EPM. Todas foram avaliadas por meio de anamnese padronizada, exame físico e estudo urodinâmico (fluxometria, cistometria e perfil pressórico uretral).

A anamnese consistiu em questionário padrão no qual se inquiriu sobre a perda de urina aos minimos, médios ou grandes esforços, bem como outros sintomas uroginecológicos (urgência miccional, urge-incontinência urinária, disúria, hematúria, polaciúria, repleção vesical, força para iniciar a diurese, esvaziamento incompleto, enurese noturna e perda constante de urina).

O exame ginecológico foi realizado pela equipe de pós-graduandos do setor, em posição ginecológica e ortostática, sendo solicitadas a tossir, assim observando a perda ou não de urina pelo orifício uretral externo.

O diagnóstico das distopias genitais baseouse na identificação da procidência das paredes vaginais e do colo uterino, espontaneamente e/ ou sob tração. Classificou-se o prolapso de uretra e bexiga nos seguintes graus: 0: posição normal; $1^{\circ}$ grau: ao esforço solicitado, há descida da parede vaginal anterior até o terço inferior da vagina; $2^{\circ}$ grau: quando ao esforço, a parede vaginal atinge o intróito vaginal; $3^{\circ}$ grau, quando ultrapassa o intróito ${ }^{15}$.

Já o prolapso uterino foi classificado em de $1^{\circ}, 2^{\circ}$ e $3^{\circ}$ graus $^{16}$. No de $1^{\circ}$ grau, o colo pinçado e tracionado atinge o terço inferior da vagina, mas não ultrapassa o intróito vaginal; no de $2^{\circ}$ grau apenas o colo ou parte do corpo, pinçados e tracionados, exteriorizam-se através do intróito, e no de $3^{\circ}$ grau, o colo e todo o corpo ultrapassam a fenda vulvar.

O perfil uretral foi realizado utilizando-se cateter de fluxo número 8 e os parâmetros avaliados foram a pressão máxima de fechamento uretral e o comprimento funcional da uretra.

Utilizou-se a análise estatística dos dados amostrais, por meio da determinação interna de um teste diagnóstico, para calcular a sensibilidade, especificidade e os valores preditivos positivo e negativo do sinal clínico ${ }^{17}$.

\section{Resultados}

A média de idade foi de 51 anos (19-80), sendo que 61 encontravam-se no menacme $(53,5 \%)$ e $53(46,5 \%)$ na pós-menopausa. Destas, 10 (18,8\%) faziam uso da terapia de reposição hormonal. Do total de pacientes, 25 (21,9\%) haviam se submetido a cirurgias prévias para incontinência. A paridade média foi de 4,2 filhos (0-12). 
A Tabela 1 evidencia as características encontradas no exame físico com relação às distopias genitais. Observa-se que a maioria das pacientes tinham cistocele de $1^{\circ}$ ou $2^{\circ}$ grau, leve a moderada retocele e ausência de prolapso uterino.

Tabela 1 - Classificação e características das distopias genitais encontradas no exame físico.

Cistocele

$\begin{array}{lc}\text { ausente } & 27(23,7 \%) \\ 1^{\circ} \text { grau } & 36(31,6 \%) \\ 2^{\circ} \text { grau } & 40(35,0 \%) \\ 3^{\circ} \text { grau } & 11(9,7 \%) \\ \text { tocele } & \\ \text { ausente } & 32(18,1 \%) \\ \text { leve } & 47(41,2 \%) \\ \text { moderada } & 31(27,2 \%) \\ \text { grave } & 4(3,5 \%) \\ \text { lapso uterino } & \\ \text { ausente } & 93(81,6 \%) \\ 1^{\circ} \text { grau } & 13(11,4 \%) \\ 2^{\circ} \text { grau } & 8(7,0 \%) \\ 3^{\circ} \text { grau } & 0(0,0 \%)\end{array}$

A prevalência das queixas clinicas uroginecológicas e sua correlação com o diagnóstico urodinâmico final podem ser visualizadas na Tabela 2 . A perda aos esforços como única queixa foi observada em 41 mulheres $(36,0 \%)$, sendo que nenhuma delas apresentava bexiga instável. Do mesmo modo, 13 com queixa de urgência/urge-incontinência isoladas não apresentavam IUE à avaliação urodinâmica $(11,4 \%)$. Contudo, observamos que $60(52,6 \%)$ das pacientes tinham sintomas mistos, constituindo a maioria na população estudada.

Tabela 2 - Prevalência das queixas clínicas uroginecológicas e sua correlação com os achados urodinâmicos.

\begin{tabular}{ccccc}
\hline & \multicolumn{4}{c}{ Diagnóstico Urodinâmico } \\
& IUE & ID & IUM & Normal \\
\hline Perda aos esforços isolada & 34 & 0 & 2 & 5 \\
$41(36,0 \%)$ & $(83,0 \%)$ & $(0 \%)$ & $(4,9 \%)$ & $(12,1 \%)$ \\
Urgência/urge-incontinência & 0 & 5 & 1 & 7 \\
isolada & & & & \\
$13(11,4 \%)$ & $(0 \%)$ & $(38,5 \%)$ & $(7,7 \%)$ & $(53,8 \%)$ \\
Sintomas mistos & 25 & 10 & 10 & 15 \\
$60(52,6 \%)$ & $(41,6 \%)$ & $(16,7 \%)$ & $(16,7 \%)$ & $(25,0 \%)$ \\
\hline
\end{tabular}

IUE - Incontinência urinária de esforço

ID - Instabilidade do detrusor

IUM - Incontinência urinária mista
A correlação entre o sinal clínico de perda urinária e o estudo urodinâmico está resumida na Tabela 3. Incluiu-se em outros diagnósticos a instabilidade do detrusor, as disfunções miccionais e a hipersensibilidade vesical. O sinal clínico de perda urinária ao esforço estava presente em 50 mulheres $(43,8 \%)$, sendo que 41 (82\%) tinham o componente de esforço no estudo urodinâmico. Observamos, ainda, que 9 pacientes com este sinal clinico não tinham IUE (18\%). Entre as 64 pacientes com o sinal ausente $(56,2 \%)$, o componente de esforço foi observado em 30 (46,9\%). Os valores preditivos positivo e negativo do sinal clínico para o diagnóstico de IUE, neste grupo estudado, foram de $82 \%$ e $53,1 \%$, respectivamente.

Tabela 3 - Correlação entre 0 sinal clínico de perda urinária e 0 estudo urodinâmico.

\begin{tabular}{lccc}
\hline & \multicolumn{3}{c}{ Diagnóstico urodinâmico } \\
Sinal clínico & IUE & IUE + outro diagnóstico & Sem IUE \\
\hline Presente & 35 & 6 & 9 \\
$(n=50)$ & $(70 \%)$ & $(12 \%)$ & $(18 \%)$ \\
Ausente & 23 & 7 & 34 \\
$(n=64)$ & $(35,9 \%)$ & $(11 \%)$ & $(53,1 \%)$ \\
\hline IUE - Incontinência urinária de esforço & & \\
Sensibilidade: $57,7 \%$ & & \\
Especificidade: $79,1 \%$ & & \\
Valor preditivo positivo : $82 \%$ & & \\
Valor preditivo negativo: $53,1 \%$ &
\end{tabular}

\section{Discussão}

A avaliação inadequada e o diagnóstico incorreto da etiologia da incontinência urinária têm múltiplas conseqüências, sendo a mais séria a indicação de cirurgias inapropriadas ou mesmo desnecessárias. Procedimentos cirúrgicos sucessivos tem menores indices de sucesso progressivamente, além de maior risco cirúrgico e maior taxa de complicações pós-operatórias. É largamente aceito que o melhor procedimento anti-incontinência, em termos de sucesso, é o primeiro ${ }^{18}$.

Outro fator importante, nos dias atuais, são os custos financeiros e humanos, além das implicações médico-legais de um procedimento cirúrgico desnecessário ou mesmo incorreto.

Montz e Stanton ${ }^{19}$ afirmaram que uma em nove mulheres pode ser operada desnecessariamente quando o diagnóstico se baseia apenas em dados de anamnese e nos resultados do $Q$-tip test, alertando que, desse modo, o tratamento será empírico e o êxito cirúrgico será baixo.

De acordo com Jensen et al. ${ }^{18}$, a história clínica apresenta baixos valores preditivos do diagnóstico urodinâmico final. Observaram que pacien- 
tes com queixa de perda urinária ao esforço podem ter, na avaliação urodinâmica, o componente de esforço, contrações não inibidas ou mesmo a coexistência de ambos. A queixa de perda urinária ao esforço tem sensibilidade de 0,90 e baixa especificidade $(0,55)$, resultando em elevados indices de falso-positivo. Baseado nestes dados, a confiança somente em dados da história clínica pode levar a erro diagnóstico em até $25 \%$ dos casos.

Embora Farrar et al. ${ }^{4}$ tivessem diagnosticado bexigas instáveis em apenas 3,6\% das pacientes com o sintoma puro de perda ao esforço, Haylen et al. ${ }^{12}$ observaram que este, como queixa única, é muito incomum. Na população estudada, encontramos os sintomas mistos como sendo os mais prevalentes $(52,6 \%)$ e não encontramos nenhuma paciente com instabilidade do detrusor entre aquelas com sintoma único de perda aos esforços. Da mesma forma, aquelas com queixa de urgência/urge-incontinência isoladas não apresentavam IUE, à avaliação urodinâmica.

A instabilidade do detrusor associada a IUE é importante fator de risco na predição do sucesso terapêutico e deve ser proposto tratamento clinico antes da cirurgia para este grupo de pacientes. Alterações na micção também são achados relevantes, uma vez que estas mulheres têm risco aumentado para retenção urinária no pós-operatório ${ }^{13}$.

$\mathrm{Na}$ predição de hiperatividade vesical, os relatos da história estão associados a baixas sensibilidade $(0,73)$ e especificidade $(0,52)$. Baseando-se somente em dados da história, o erro diagnóstico pode chegar a até $45 \%$. Da mesma forma, para a incontinência urinária mista a história possui baixa sensibilidade $(0,484)$ e especificidade $(0,656)$, com valores preditivos de acerto em apenas $25 \%$ dos casos $^{18}$.

Há que se lembrar, também, que a quantificação clínica da perda urinária é subjetiva, já que se baseia em informações prestadas pela paciente, e o impacto dessa perda é muito variável, conforme dados culturais, socioeconômicos e raciais. Haylen et al. ${ }^{12}$ concluíram que a demonstração clínica da perda urinária ao esforço estava presente em apenas $41 \%$ dos casos com o diagnóstico final de IUE, pura ou mista. Além disso, 32\% das pacientes com este sinal tinham outro diagnóstico que não a IUE somente.

Carey et al. ${ }^{13}$ confirmam tais observações ao avaliar a eficácia do sinal clínico de perda aos esforços comparado ao estudo urodinâmico em 863 pacientes. Concluíram que o primeiro está presente em apenas $54,2 \%$ das 574 mulheres com diagnóstico final de IUE. Além disso, 38\% tinham outro diagnóstico adicional, como instabilidade vesical. Observaram que o sinal clinico tem valor preditivo positivo de $91 \%$ e valor preditivo negativo de $50 \%$.

Mesmo com elevados valores preditivos po- sitivos em ambos os estudos, nenhum dos trabalhos tem elementos que indiquem a possibilidade de diferenciação da incontinência urinária de esforço por defeito esfincteriano, o que seria importante na escolha do tratamento cirúrgico.

Em contraposição, Weidner et al. ${ }^{14}$ demonstraram que o sinal clínico tem valor preditivo positivo de $68,2 \%$ e valor preditivo negativo de $88,6 \%$, indicando que a ausência de perda urinária ao exame clínico é mais útil na exclusão do diagnóstico que na sua confirmação.

Em nosso estudo, o sinal clínico de perda urinária ao esforço estava presente em 43,8\% mulheres, sendo que $82 \%$ tinham o componente de esforço ao estudo urodinâmico. Observamos, ainda, que $18 \%$ das pacientes com este sinal clinico tinham exame urodinâmico normal ou outro diagnóstico que não a IUE. O mesmo estava ausente em $56,2 \%$ dos casos, sendo que o componente de esforço foi observado em 46,9\% destas pacientes. Os valores preditivos positivos e negativo do sinal clínico no diagnóstico de IUE, neste grupo estudado, foram de 82 e 53,1\%, respectivamente.

O objetivo da avaliação urodinâmica é identificar as causas específicas dos sintomas das pacientes, seja a incontinência urinária, a disfunção miccional ou sintomas irritativos do trato urinário ${ }^{20}$, além de fornecer dados para orientar o correto tratamento, seja ele cirúrgico ou não. O diagnóstico final é o resultado de uma contínua interação entre a paciente e o examinador, sendo de importância fundamental a interpretação dos dados e a separação de informações relativas a artefatos ${ }^{21}$.

Baseando-se nestes dados, acreditamos que a história clínica associada ao exame físico têm importância no manejo da incontinência urinária; porém, não devem ser utilizados como único método diagnóstico. Seu valor recai sobre o rastreamento na identificação de pacientes que requerem investigação posterior. Os testes objetivos estão disponiveis e devem ser utilizados em conjunto com os dados clínicos, permitindo um diagnóstico preciso e uma terapia apropriada.

\section{ABSTRACT}

Purpose: to analyze the prevalence of urogynecological symptoms and their relationship with final urodynamic diagnosis, and to compare the clinical sign of stress urinary incontinence with urodynamic diagnosis.

Methods: a total of 114 patients were included in a retrospective study from June 2000 to January 2001. All patients were evaluated through medical interview, physical examination and urodynamic study. They were classified according to clinical symptom, presence of clinical sign of 
urine loss and urodynamic study. The data analysis was performed using a test to determine sensitivity, specificity, and positive and negative predictive values.

Results: the mean age was 51 years (19-80), 61 patients (53.5\%) were in menacme and 53 (46.5\%) in postmenopausal stage. Ten (18.8\%) were using hormone replacement therapy and $25(21.9 \%)$ had been submitted to surgery for incontinence. The isolated clinical symptom of urine loss was reported in $41(36.0 \%)$ patients, the isolated urgency/urgencyincontinence in $13(11.4 \%)$ and mixed symptoms in $60(52.6 \%)$. In the urodynamic study, of all patients with symptom of isolated urine loss, 34 (83\%) had stress urinary incontinence (SUI), no patient had detrusor instability (DI), 2 (4.9\%) had mixed incontinence (MI) and 5 (12.1\%) had a normal result. Of all patients with isolated urgency/urgency-incontinence, in the urodynamic study, none had SUI, 5 (38.5\%) had ID, 1 (7.7\%) had MI and 7 (53.8\%) had a normal result. Of the patients with mixed symptoms, we identified, on the urodynamic evaluation, 25 (41.6\%) who had SUI, 10 (16.7\%) ID, 10 (16.7\%) MI and 15 (25.0\%) a normal result. The clinical sign of urine loss was identified in $50(43.9 \%)$ patients. A total of 35 (70\%) had SUI on urodynamic study, 6 (12\%) had SUI and another diagnosis and $9(18 \%)$ did not have SUI. Urine loss was absent in 64 (56.1\%) women. Of those 23 (35.9\%) had SUI on urodynamic study, 7 (11\%) had SUI and another diagnosis and 34 (53.1\%) did not have SUI.

Conclusions: clinical history and physical examination are important in the management of urinary incontinence, although they should not be used as the only diagnostic method. Objective tests are available and should be used together with clinical data.

KEY WORDS: Urinary incontinence. Clinical examination. Urodynamic study.

\section{Referências}

1. International Continence Society. Committee on Standardisation of Terminology. The standardisation of terminology of lower urinary tract function. In: Ostergard DR, Bent AE, editors. Urogynecology and urodynamics: theory and practice. 3rd ed. Baltimore: Williams \& Wilkins; 1991. p.545-62.

2. Kujansuu E. Patient history in the diagnosis of urinary incontinence and determining the quality of life. Acta Obstet Gynecol Scand Suppl 1997; 166:15-8.

3. Julian TM. Physical examination and pretreatment testing of incontinent woman. Clin Obstet Gynecol 1998; 41:663-71.

4. Farrar DJ, Whiteside CG, Osborne JL, TurnerWarwick RT. A urodynamic analysis of micturition symptoms in the female. Surg Gynecol Obstet 1975; 141:875-81.

5. Thiede HA, Saini VD. Urogynecology: comments and caveats. Am J Obstet Gynecol 1987; 157:563-8.

6. Versi E, Cardozo L, Anad D, Cooper D. Symptom analysis for the diagnosis of genuine stress incontinence. Br J Obstet Gynaecol 1991; 98:815-9.
7. De Muylder X, Claes H, Neven. P, De Jaegher K. Usefulness of urodynamic investigations in female incontinence. Eur J Obstet Gynecol Reprod Biol 1992; 44:205-8.

8. Summitt RL Jr, Stovall TG, Bent AE, Ostergard DR. Urinary incontinence: correlation of history and brief office evaluation with multichannel urodynamic testing. Am J Obstet Gynecol 1992; 166:1835-44.

9. Haeusler G, Hanzal E, Joura E, Sam C, Koelbl H. Differential diagnosis of detrusor instability and stress incontinence by patient history: The Gaudenz-Incontinence-Questionnaire revisited. Acta Obstet Gynecol Scand 1995; 74:635-7.

10.Stamey TA. Endoscopic suspension of the vesical neck for urinary incontinence in females. Report on 203 consecutive patients. Ann Surg 1980; 192:465-71.

11.Videla FL, Wall LL. Stress incontinence diagnosed without multichannel urodynamic studies. Obstet Gynecol 1998; 91:965-8.

12. Haylen BT, Sutherst JR, Frazer MI. Is the investigation of most stress incontinence really necessary? Br J Urol 1989; 64:147-9.

13. Carey MP, Dwyer PL Glenning PP. The sign of stress incontinence - should we believe what we see? Aust N Z J Obstet Gynaecol 1997; 37:436-9.

14.Weidner AC, Myers ER, Visco AG, Cundiff GW, Bump RC. Which women with stress incontinence require urodynamic evaluation? Am J Obstet Gynecol 2001; 184:20-7.

15.Juma S, Little NA, Raz S. Evaluation of stress urinary incontinence. In: Buchsbaum HJ, Schimidt JD, editors. Gynecologic and Obstetric Urology. $3^{\text {rd }}$ ed. Philadelphia: W.B. Saunders; 1993. p.251-63. (consta no texto após a 14)

16.Beecham CT. Classification of vaginal relaxation. Am J Obstet Gynecol 1980; 136:957-9.

17.Drummond JP, Silva E. Conceitos básicos em bioestatística e em epidemiologia clínica. In: Drummond JP, Silva E, editores. Medicina Baseada em Evidências. $1^{a}$ ed. São Paulo: Atheneu; 1998. p.37-40.

18.Jensen JK, Nielsen FR Jr, Ostergard DR. The role of patient history in the diagnosis of urinary incontinence. Obstet Gynecol 1994; 83:904-10.

19. Montz FJ, Stanton SL. Q-tip test in female urinary incontinence. Obstet Gynecol 1986; 67:258-60. (consta no texto após a 17)

20.Jensen JK. Urodynamic evaluation. In: Ostergard DR, Bent AE, editors. Urogynecology and Urodynamics: theory and practice. 3rd ed. Baltimore: Williams \& Wilkins; 1996. p.115-49.

21.Wall LL, Norton PA. Practical urodynamics. In: Wall LL, Norton PA, editors. Practical Urogynecology. Baltimore: Williams \& Wilkins; 1993. p.83-124.

Recebido em: 06/11/01 Aceito com modificações em: 25/01/02 